\title{
Geographic Dependence of the Solar Irradiance Spectrum at Intermediate to High Frequencies
}

\author{
Golan Bel ${ }^{1,2}$ and M. M. Bandi $\oplus^{3, *}$ \\ ${ }^{1}$ Department of Solar Energy and Environmental Physics, Blaustein Institutes for Desert Research, Ben-Gurion \\ University of the Negev, Sede Boqer Campus, Beersheba 84990, Israel \\ ${ }^{2}$ Center for Nonlinear Studies (CNLS), Theoretical Division, Los Alamos National Laboratory, Los Alamos, \\ New Mexico 87545, USA \\ ${ }^{3}$ Nonlinear and Non-equilibrium Physics Unit, Okinawa Institute of Science and Technology Graduate University, \\ Okinawa 904-0495, Japan
}

(Received 28 May 2019; revised manuscript received 16 July 2019; published 16 August 2019)

\begin{abstract}
Temporal fluctuations in the solar irradiance are important for renewable-energy harvesting, as well as for climate and ecological-systems analysis. Environmental studies usually focus on slow fluctuations of the solar irradiance, assuming that fast fluctuations are averaged out, while studies of solar power generation emphasize the role of faster fluctuations, due to their effect on grid stability. Several analyses have reported a power-law dependence of the generated-power spectrum for frequencies ranging from $1 / \mathrm{min}$ to 1 /year. However, both the origin of this power law and its geographic dependence are not fully understood, an issue of significance when one considers smoothing of solar-photovoltaic-power fluctuations by combining geographically distributed generation sources. Here we show that the power law appears in the global and direct components of the solar radiation and not only in the generated power. We also show that the exponent of the power law has a clear latitudinal dependence. At most locations, the spectral power-law dependence of the intermediate $(1 /$ day $<f<1 / \mathrm{h})$ frequencies is different from that of the high $(f>1 / \mathrm{h})$ frequencies. Using a simple model, we explain the origin of the power-law spectra and the latitudinal dependence. The implications of the power law for photovoltaic power generation and grid stability are discussed. We also suggest that the analysis of solar power generation should consider the power spectrum of the clear-sky irradiance at the location of interest in order to separate atmospheric effects from geographical ones.
\end{abstract}

DOI: 10.1103/PhysRevApplied.12.024032

\section{INTRODUCTION}

The solar irradiance is directly or indirectly responsible for almost all life and energy processes on the Earth. It plays an important role in the analysis and modeling of climate [1,2] and of ecological systems [3-5]. Usually only slow variations in the solar irradiance are deemed important for climatic and ecological processes, and fluctuations on subdaily timescales are averaged out, although cases to the contrary do exist [6-9]. In solar-energy harvesting and, in particular, for solar photovoltaic power (PV) production, relatively faster fluctuations at frequencies $f>$ $1 /$ day are of greater importance [10] because they fall into the range within which grid responses and consumer

\footnotetext{
*bandi@oist.jp

Published by the American Physical Society under the terms of the Creative Commons Attribution 4.0 International license. Further distribution of this work must maintain attribution to the author(s) and the published article's title, journal citation, and DOI.
}

load fluctuations occur. Since the solar irradiance varies with location on the Earth, particularly due to changes in the angle of incidence and the dependence of diurnal duration on latitude due to the Earth's obliquity (the axial tilt relative to the orbital plane), it impacts on all the abovementioned processes. Therefore, the importance of understanding the geographic dependence of the spectral characteristics of the solar irradiance cannot be overemphasized.

Specifically, in the context of solar photovoltaic power, several studies have reported that the spectrum of solarphotovoltaic-power fluctuations decays with a power law [11-18]. The reported power-law exponents exhibit considerable variability, whose origin is yet to be explained. Further interest lies in the geographic smoothing of power fluctuations when the outputs from geographically distributed photovoltaic plants are summed by a grid. Here, we study the geographic dependence of the solar irradiance spectrum at various locations on the Earth using data generated from an open-source Ineichen-Perez clear-sky model (CSM) [19,20], which we first compare against a 
measured spectrum for a specific location (Sede Boqer, Israel, coordinates $30.852310,34.780586$, altitude $490 \mathrm{~m}$ above sea level). The analysis of spectra from real measurements is complicated by particularities such as local topographic features, strong fluctuations from cloud passage that act as multiplicative noise, etc. Calculated CSM spectra help separate these dependencies and allow one to focus on spectral features that are dependent on the geographic location alone.

Our analysis concludes that both measured and calculated (using a bare CSM without accounting for topography or atmospheric effects such as clouds and turbidity) spectra for the solar irradiance exhibit a power-law dependence. The appearance of the power-law dependence in the spectrum of the CSM shows that this dependence is not limited to photovoltaic power production or cloud occlusion. More specifically, the modulation at various frequencies in the solar irradiance signal results in a continuous spectrum with a power-law dependence on the frequency $(f)$, which in most locations has two separate slopes for intermediate $(1 /$ day $<f<1 / \mathrm{h})$ and high $(f>$ $1 / \mathrm{h}$ ) frequencies. In fact, Madanchi et al. [21] have already reported an observed change in the slope of the spectral power-law decay around frequencies corresponding to $f \sim$ $1 / 7$ min and $f \sim 1 / 90 \mathrm{~min}$. Although our change in the slope around $f \sim 1 / \mathrm{h}$ is close to their $f \sim 1 / 90$ min mark, our data cannot resolve the $f \sim 1 / 7$ min slope change because our sampling resolution is limited to $f=1 / \mathrm{min}$.

The power-law exponent varies systematically with geographic location (in particular, with latitude). We employ a minimal toy model to show that a slow seasonal variation in daylight duration is sufficient to explain the appearance of the power-law dependence in the solar irradiance spectrum. Since daylight duration and its seasonal variation are location-dependent, our model also explains the geographic dependence of the spectrum's power-law exponent. We close with a discussion of the significance of the abovementioned results in the specific context of solar photovoltaic production, namely, that power-law exponents for measured photovoltaic-power spectra should be compared with solar-irradiance spectral exponents to provide a sound comparative basis for different locations. In emphasizing the importance of the power-law exponent at a given location, we make specific recommendations for operators of solar PV farms to consider, both prior to development of the farm and during its operation, and for independent system operators to consider from the standpoint of geographic smoothing of fluctuations.

\section{METHODS}

Solar radiation comprises three different components [22-25]: The direct (beam) radiation is the intensity of radiation traveling in a straight line from the Sun to a measuring device. The diffuse radiation is the intensity of radiation scattered by clouds, local topographic features, etc. and is isotropic. The global radiation is the direct radiation multiplied by the cosine of the angle between the measuring plane (assumed to be parallel to the Earth's surface) and the Sun, plus the diffuse radiation component. The global radiation is, therefore, not independent of the other two. We focus on the global and direct radiation components in this study.

The solar radiation incident on the Earth exhibits modulation over timescales far greater than the diurnal oscillation timescale ( $f=1 /$ day), from seasonal/annual periods extending to hundreds of millennia (Milankovitch cycles $[26,27])$. Therefore, the solar-insolation time series does not approach stationarity over any reasonable measurement time period, thereby invalidating the application of time-domain methods. Consequently, strictly spectral methods are needed, which is why our analysis is based on the power spectrum of solar radiation. In addition to the spectral power-law exponent, alternative methods involving the Hurst and Renyi exponents exist, but they can only be extracted in the time domain. A detrended fluctuation analysis (DFA) [28] certainly helps to normalize the fluctuations [29] and permits a time-domain treatment [21,30], but it expressly does so by removing the daylight duration modulations so that fluctuations caused by cloud occlusion may be analyzed in a principled manner. In the current paper, we focus purely on variations of the solar irradiance without cloud effects; therefore, DFA is not relevant. Be that as it may, we draw the reader's attention to the fact that the Hurst exponent, $H$, is related to the spectral power-law exponent $\zeta$ through a simple linear relation: $\zeta=1+2 H$. With this definition, given that a higher $H$ value indicates a smooth signal, we observe that the bare solar-irradiance signal transitions from a smoother to a rougher signal as one proceeds from the equator toward the poles, as we show later.

The power spectrum presented here has the dimensions of the square of the Fourier transform of the temporal radiation intensity, namely, units of $\mathrm{W}^{2} \mathrm{~m}^{-4} \mathrm{~min}^{2}$. The Fourier transform, or more precisely series, is defined for the discrete signals that we analyze as

$$
\begin{aligned}
\tilde{R}\left(f_{n}\right) & =\sum_{k=1}^{N} W_{N}^{k n} R\left(t_{k}\right), \\
W_{N} & \equiv \exp (-2 \pi i / N) .
\end{aligned}
$$

Here $N$ is the length of the time series, and the discrete frequencies are given by $f_{n} \equiv n /(N \Delta t)$, where $n \in[0, N / 2]$ and $\Delta t$ is the time interval. We use a year-long time series with a 1-min resolution for both measured and calculated radiation. The calculated radiation is based on the CSM $[19,20]$, which calculates the radiation based on the time, location, altitude, and, if required, also air turbidity using empirical values for the location-dependent Linke turbidity 


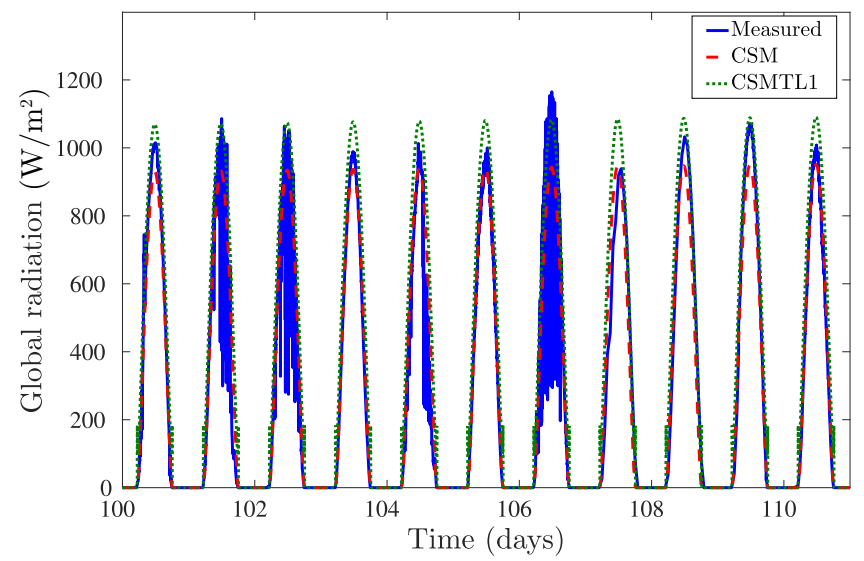

FIG. 1. Measured (blue solid line), CSM (red dashed line), and CSMTL1 (dotted red line) global component of the solar radiation in Sede Boqer during days $100-110$ of 2009 . The days were chosen to illustrate the fact that the CSM does not account for clouds and specific atmospheric conditions. However, it does capture the daily cycle correctly and also the annual amplitude variation (not shown).

index [31,32], which varies monthly (according to the calendar month). The CSM does not account for slow changes in the Earth's orbit, for intrinsic fluctuations in the solarirradiance intensity, or for specific atmospheric conditions at a given time and location. A report on clear-sky models found the CSM to have excellent performance for a range of geographic latitudes [33]. Calculations employing the CSM with no turbidity (Linke turbidity $\mathrm{TL}=1$ ) are referred to as CSMTL1. The advantage of using the CSM is that it allows one to calculate the solar irradiance without the need to provide details regarding the atmospheric conditions, which are not known at all locations. In Fig. 1, we present the global component of the solar radiation for days 100-110 of 2009. The different curves correspond to the measured and the CSM- and CSMTL1-calculated radiation. As can be seen, the model and measurements agree on the duration of the daily cycle. However, the amplitudes differ because the CSM and CSMTL1 do not consider the clouds that were present on those days. The CSM does capture the annual variation of the solar-radiation amplitude. We also test the model for several locations around the equatorial Pacific, for which we find measurement data with high temporal resolution, and find similar agreement.

In what follows, we rely extensively on power-law fits of the spectra obtained. By this, we mean that if the power spectral density $\operatorname{PS}(f)$ varies with frequency $f$ with a functional form $\operatorname{PS}(f)=A f^{\zeta}$, we say that the power spectrum exhibits a power law with an exponent $\zeta$, and the prefactor $A$ captures the magnitude of the spectrum (subtracting the mean from the original signal ensures that for the zero frequency, the power spectrum vanishes). If one takes the logarithm of both sides of the above equation,

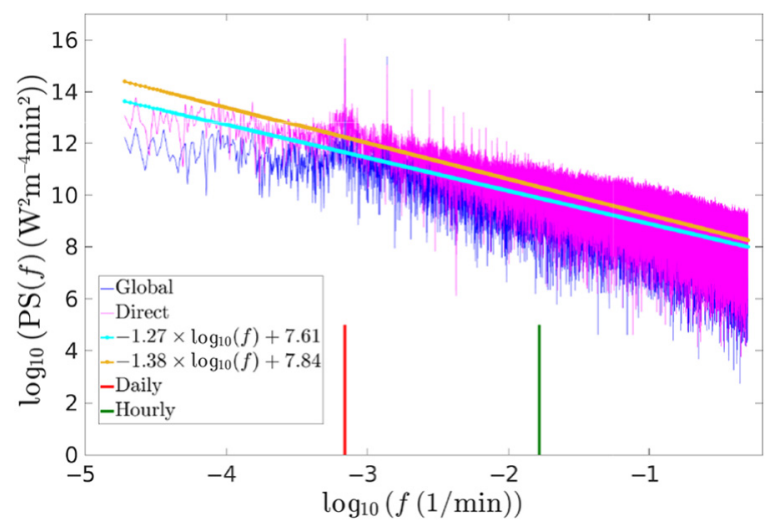

FIG. 2. Measured spectra (solid line) and fits (dots) for global (blue) and direct (pink) radiation in Sede Boqer, Israel (year 2009); the daily frequency is marked by a red vertical bar and the hourly frequency by a green vertical bar. The spectra of the two components have different power-law exponents $(\zeta$, slope of fit) but possess similar magnitudes (fit intercept). The difference between the power-law exponents $(\zeta)$ is, however, statistically significant; the $95 \%$ confidence interval for the globalcomponent exponent is $[-1.28,-1.27]$, and $[-1.39,-1.38]$ for the direct-component $\zeta$.

the spectrum can then be represented as $\log _{10}[\operatorname{PS}(f)]=$ $\log _{10}\left(A f^{\zeta}\right) \equiv \log _{10}(A)+\zeta \log _{10}(f)$. In the following, we plot $\log _{10}[\mathrm{PS}(f)]$ versus $\log _{10}(f)$ when presenting spectra so that the slope of the plot yields the power-law exponent $\zeta$, and $A$ gives us the spectral magnitude. The exponent $\zeta$ is derived from a linear fit of the log-log plot without any smoothing. We find that due to broad peaks, any smoothing procedure alters the power-law exponent. Specifically, due to the higher amplitude of the daily peak relative to its higher harmonics, averaging results in an increased slope of the daily-to-hourly spectra and has just a minor effect

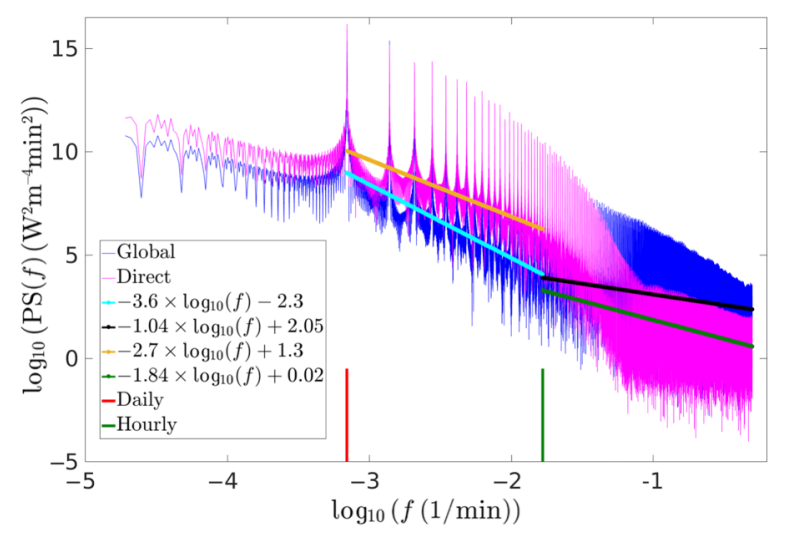

FIG. 3. Calculated (CSM) spectra (solid lines) and fits (dots) for global (blue) and direct (pink) radiation for Sede Boqer in the year 2009. The frequency range is divided into high- and intermediate-frequency ranges; the daily frequency is marked by a red vertical bar and the hourly frequency by a green vertical bar. 

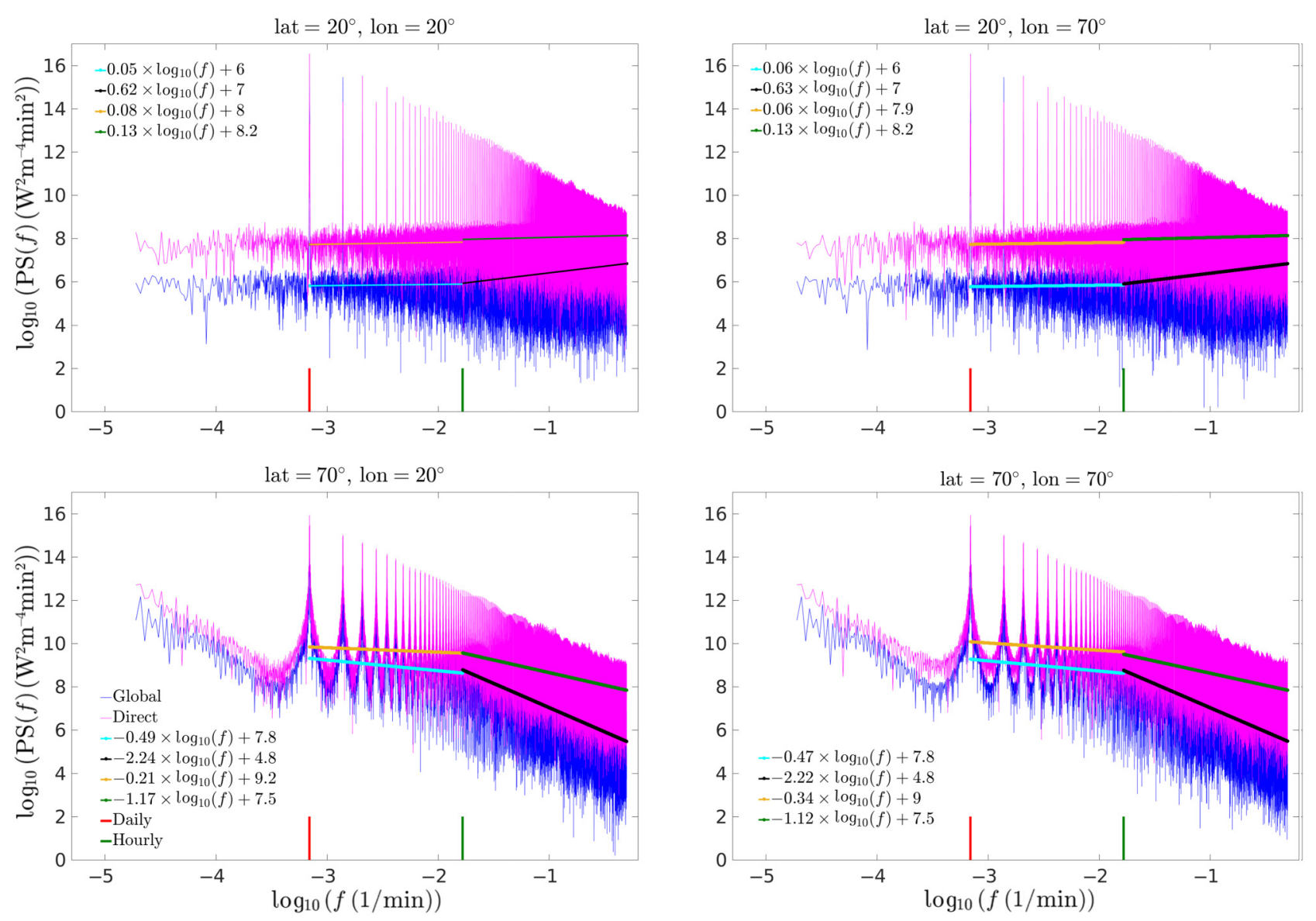

FIG. 4. Calculated (CSMTL1) global (blue) and direct (pink) radiation spectra (solid lines) for the year 2015 with zero turbidity, and their fits (dots) at four different locations with the following latitude and longitude coordinates: $\left(20^{\circ}, 20^{\circ}\right),\left(20^{\circ}, 70^{\circ}\right),\left(70^{\circ}, 20^{\circ}\right)$, and $\left(70^{\circ}, 70^{\circ}\right)$. The frequency range is divided into high and intermediate frequencies; the daily frequency is marked by a red vertical bar and the hourly frequency by a green vertical bar.

on the higher frequencies' slope. In order to avoid loss of information and bias, we derive the exponent from the spectra without averaging.

As an example, Fig. 2 presents spectra for one year's data (2009) from sensors at Sede Boqer, Israel; see also Fig. 10 for measurements during 2012. In addition to the expected peaks at the frequencies $f=1 /$ day and $f>1 /$ day (due to radiation cutoff during the hours of darkness), we observe a continuous spectrum with a reasonably fitting power-law decay for both the direct and the global components at $f>1$ day; the fits do not vary much between 2009 and 2012. In contrast, the calculated (CSM) spectra for Sede Boqer in 2009 (Fig. 3) are distinctly different (from the measured ones), particularly in the deviation from a single power-law decay over the entire frequency range observed in both components (since they are not independent of each other). A reasonable fit is achieved only when done separately for the high-frequency $(f>1 / \mathrm{h})$ and intermediate-frequency $(1 /$ day $<f<1 / \mathrm{h})$ ranges. This deviation can be understood from the fact that the CSM assumes a clear sky and monthly varying turbidity, as opposed to actual measurements, which must contend with both clouds and a faster turbidity variation.

\section{RESULTS}

Having established the spectral power law and the observable difference in it between the measured and calculated spectra, we henceforth employ only calculated (CSM and CSMTL1) spectra in our analysis. This allows us to disentangle signal variability from local topography, cloud passage, etc. and focus exclusively on the geographic dependence. To this end, we track how the exponent of the spectral power law varies with geographic location (latitude and longitude). To first demonstrate the dependence, in Fig. 4 we present CSMTL1 spectra, with the elevation set to sea level to further simplify the analysis, at four different locations. Firstly, we observe a strong variation in the spectral power-law exponent with location in both frequency regimes (high and intermediate), and, secondly, this variation exhibits a stronger dependence on the latitude than on the longitude. 


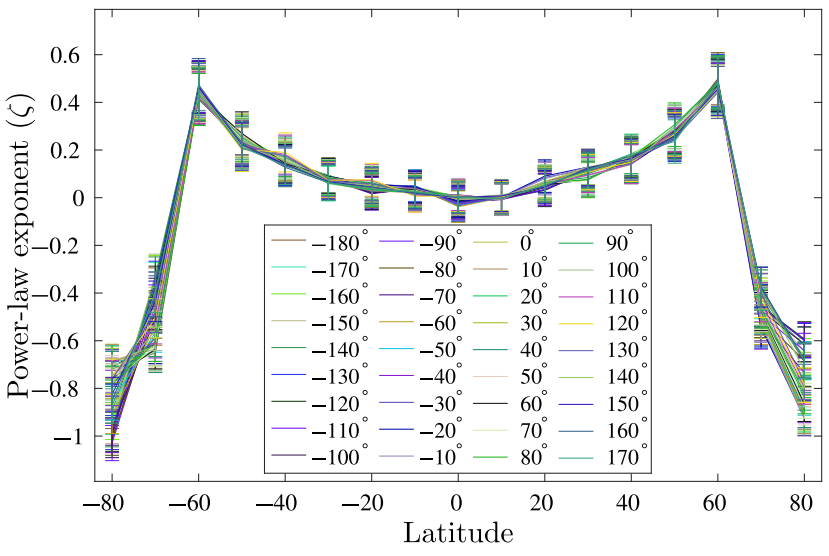

FIG. 5. Latitudinal dependence of the power-law exponent of the intermediate-frequency global radiation spectra. The powerlaw exponent is plotted against the latitude for each longitude. The latitude and the longitude vary in steps of $10^{\circ}$ in the range of latitudes from $-80^{\circ}$ to $80^{\circ}$ and longitudes from $-180^{\circ}$ to $170^{\circ}$. The different colors correspond to different longitudes as indicated. The error bars represent the $95 \%$ confidence interval of the power-law exponent.

To better understand this geographic dependence, we calculate the spectra at different coordinates for a range of latitudes $\left[-80^{\circ},+80^{\circ}\right]$ and longitudes $\left[-180^{\circ}, 170^{\circ}\right]$ at $10^{\circ}$ intervals in each coordinate. Figure 5 shows the power-law exponent $\zeta$ obtained from a fit to the global radiation spectrum for intermediate frequencies at different latitudinal coordinates, where each curve represents a different longitude. The error bars represent the 95\% confidence interval of the value derived from a linear fit to the logarithm of the power spectrum versus the logarithm of the frequency. It is apparent that the differences between the values for different longitudes are insignificant away from the poles, as is the uncertainty interval. Closer to the poles, at latitudes $-80^{\circ}$ and $-70^{\circ}$ (above the Arctic Circle), and $70^{\circ}$ and $80^{\circ}$ (below the Antarctic

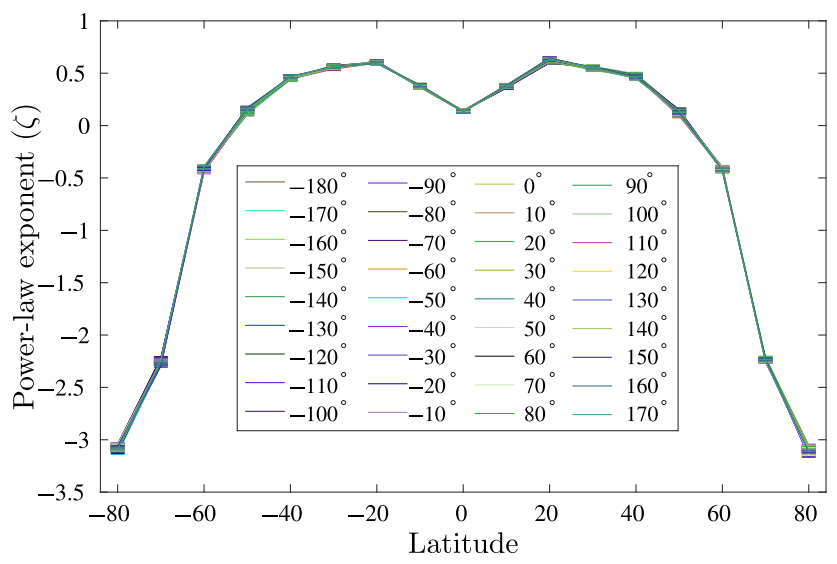

FIG. 6. Spectral power-law exponent at high frequencies for global radiation; other details are the same as in Fig. 5.

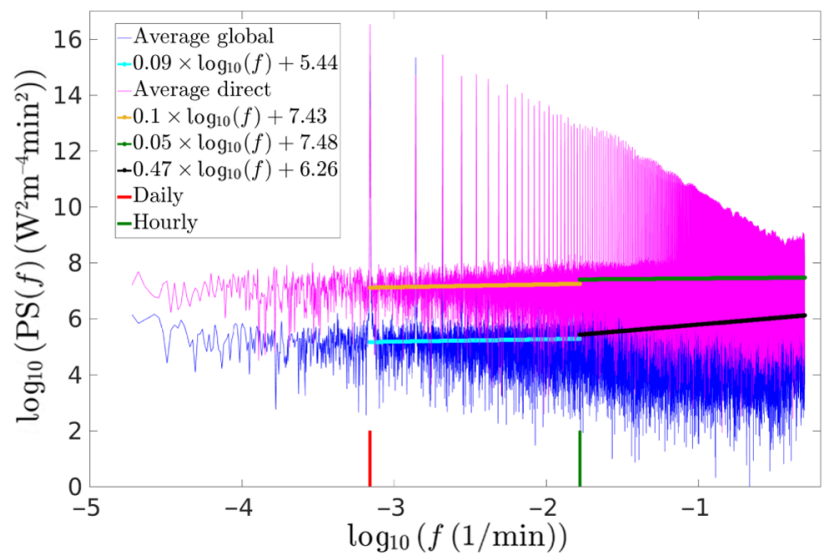

FIG. 7. Global (blue) and direct (pink) radiation spectra (solid lines) for the averaged (over four locations in the latitudinal range $29^{\circ}-32^{\circ}$ and $34^{\circ}$ longitude) radiation with fits for intermediateand high-frequency ranges.

Circle), the uncertainty is larger, yet the values for these latitudes are significantly different from those elsewhere; at these latitudes, the amplitudes of the low frequencies decay, while elsewhere they either grow slowly or are almost constant. The observed nonmonotonic dependence on latitude can be traced to the nonmonotonic seasonal variations and daylight duration. The same information is presented in Fig. 11 but versus the longitude, where each curve represents a different latitude. As expected, here too no significant longitudinal dependence is observed in the power-law exponent except near the poles; the polar longitudinal dependence is, in fact, an artifact of the power-law fit at the low-frequency end of the spectrum due to its noncontiguous nature, as shown in the lower panels of Fig. 4.

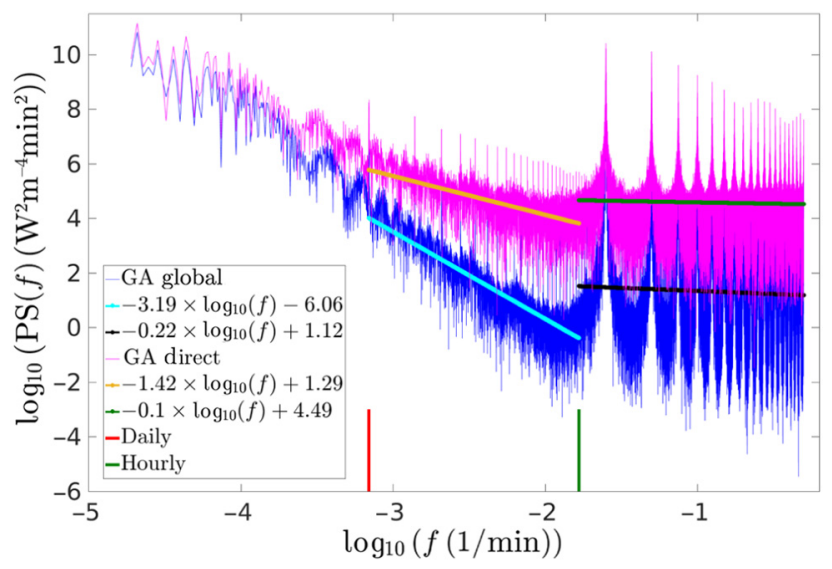

FIG. 8. Power spectra of the global and direct components of the globally averaged (GA) radiation (see text for definition of the average). 


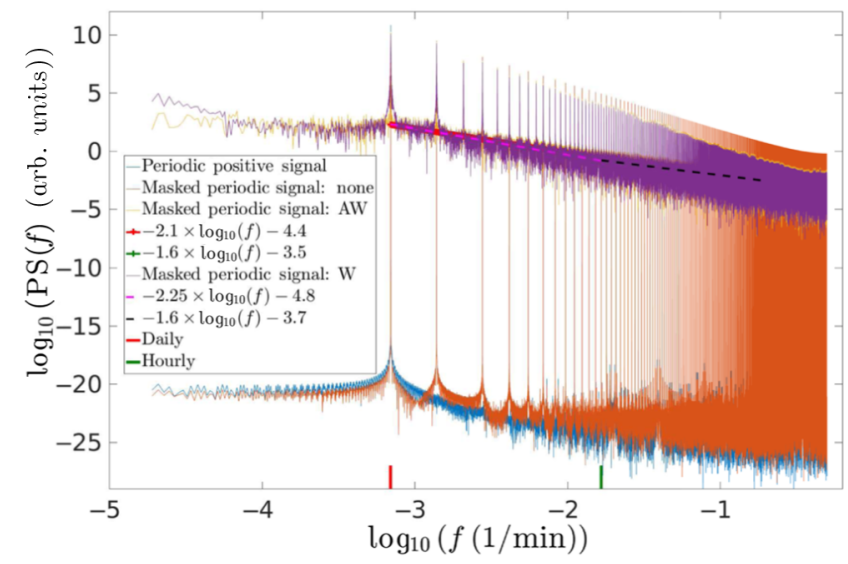

FIG. 9. Power spectra for periodic signal at daily frequency (green-yellow line), masked periodic signal with fixed $12 \mathrm{~h}$ on and off times (brown line), periodic signal with varying on time according to daylight duration at $35^{\circ} \mathrm{N}$ and daily normalized amplitude equal to 1 (blue line), and masked periodic signal with varying on time according to daylight duration at $35^{\circ} \mathrm{N}$ and also varying amplitude as determined by the mask (purple line: see text for more details). The daylight duration is updated on a daily basis during the year-long time series. The other lines present the power-law fits for the "Masked periodic signal: W" and "Masked periodic signal: AW" spectra for the intermediateand high-frequency ranges.

Figure 6 (see also Fig. 12) presents the variation of the power-law exponent versus the latitude at different longitudes for the high-frequency end of the spectra. Once again, except near the poles, the high frequencies do not decay and, in fact, even grow with frequency (a positive power-law exponent, $\zeta>0$ ). Near the poles, the spectral

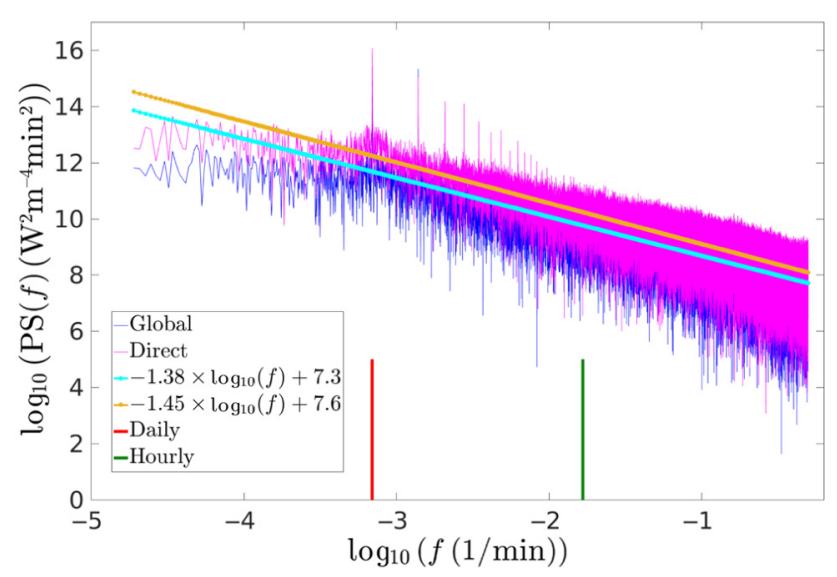

FIG. 10. Spectra for the global and direct radiation components in Sede Boqer during the year 2012. The details and notation are identical to those for Fig. 1. The 95\% confidence interval for the decay power is $[-1.39,-1.38]$ for the global and $[-1.46,-1.45]$ for the direct component. The power-law fit is similar to the one found for the radiation measured in 2009 (presented in Fig. 1), as expected.

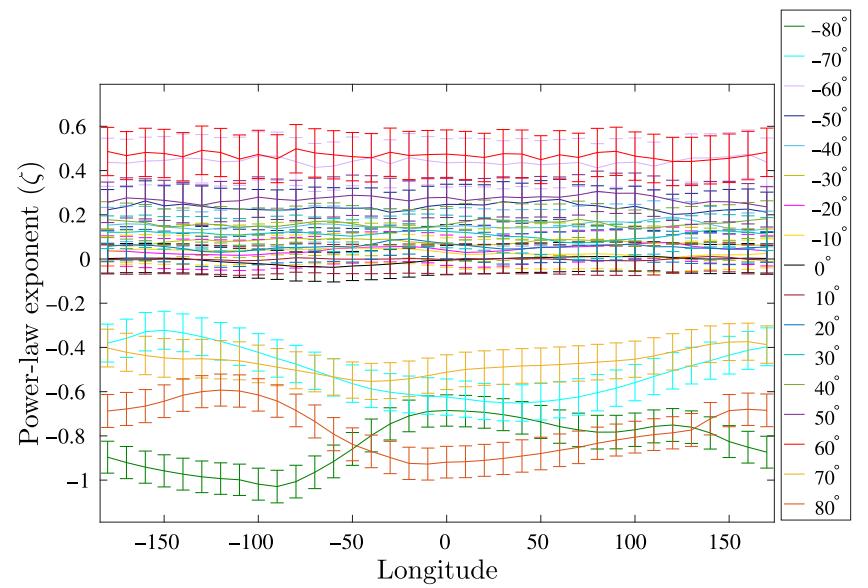

FIG. 11. Power-law exponent of the intermediate-frequencyrange global radiation spectra plotted against the longitude for each latitude. The other details are the same as in Fig. 4.

decay is sharp on account of the weak daily cycle in these regions. The high-frequency variation has no longitudinal dependence in any region, and the spread in the individual curves is indeed less than the confidence intervals of the fit. Figures 13-16 present the direct-component analog of Figs. 5-6 and 11-12 and exhibit more polar variation in the longitudinal dependence at low frequencies than for the global component. Away from the poles, the variation is low relative to the global component and almost constant with frequency, i.e., the power-law exponent is close to zero.

Having established the geographic dependence of the spectral power-law exponent, we now investigate the effect of geographic smoothing via two different averages. This smoothing analysis assumes significance particularly in the context of solar photovoltaic power production, where one is interested in the extent to which fluctuations are smoothed when power from geographically distributed

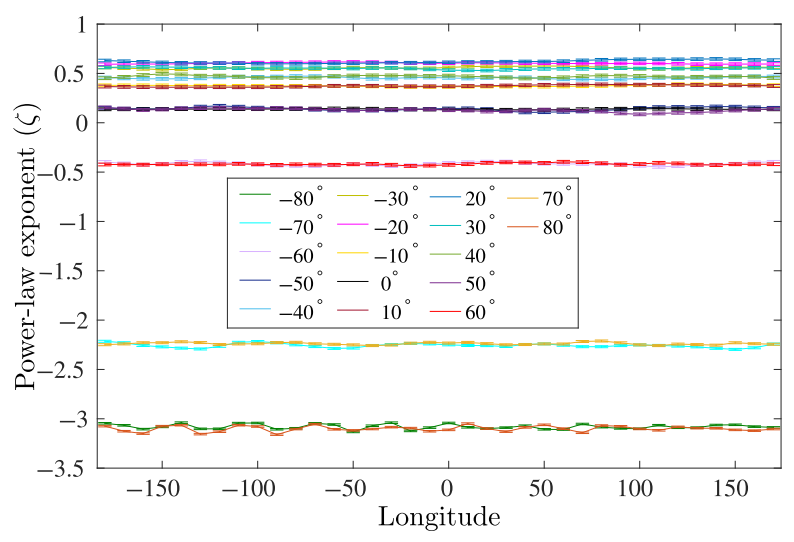

FIG. 12. Dependence of the power-law exponent of the highfrequency global radiation spectra on the longitude. Other details are the same as in Fig. 11. 


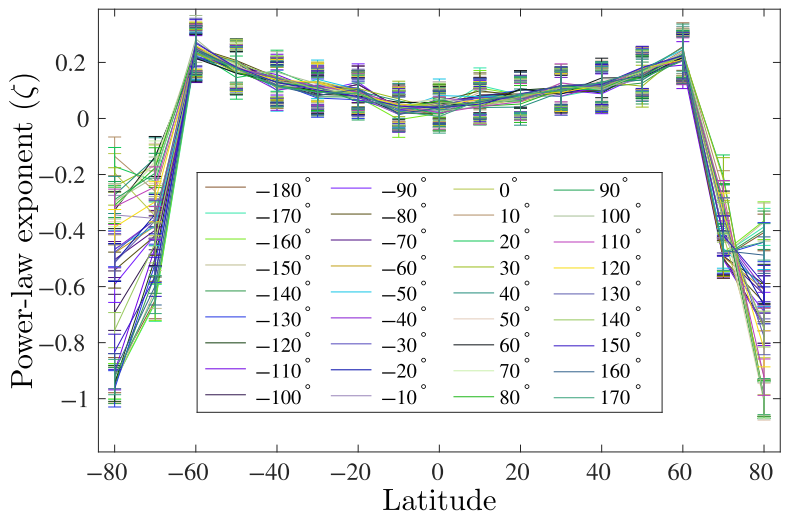

FIG. 13. Power-law exponent at intermediate frequencies for the direct radiation spectra. The power-law exponent is plotted against the latitude for each longitude. The latitude and the longitude vary in steps of $10^{\circ}$ in the range of latitudes from $-80^{\circ}$ to $80^{\circ}$ and longitudes from $-180^{\circ}$ to $170^{\circ}$. The different colors correspond to different longitudes as indicated. The error bars represent the $95 \%$ confidence interval of the power-law exponent.

photovoltaic power plants is summed in a grid. The powerlaw spectral form $f^{\zeta}$ has also been reported for wind power [29,34-36], but there the largest scales in the atmospheric flows correlate distant wind plants over hundreds of kilometers. When power from these geographically distributed plants is summed at the grid level, the fluctuations are smoothed until they reach a spectral limit of $f^{-7 / 3}$ [36]. Similarly, in solar photovoltaic power production, with the correlation length set by the Sun, all power plants become globally correlated. Considering fluctuations from cloud passage alone, a theoretical limit for geographic smoothing of photovoltaic power of $f^{-2}$ has been shown [10], but this limit does not take into account the fact that

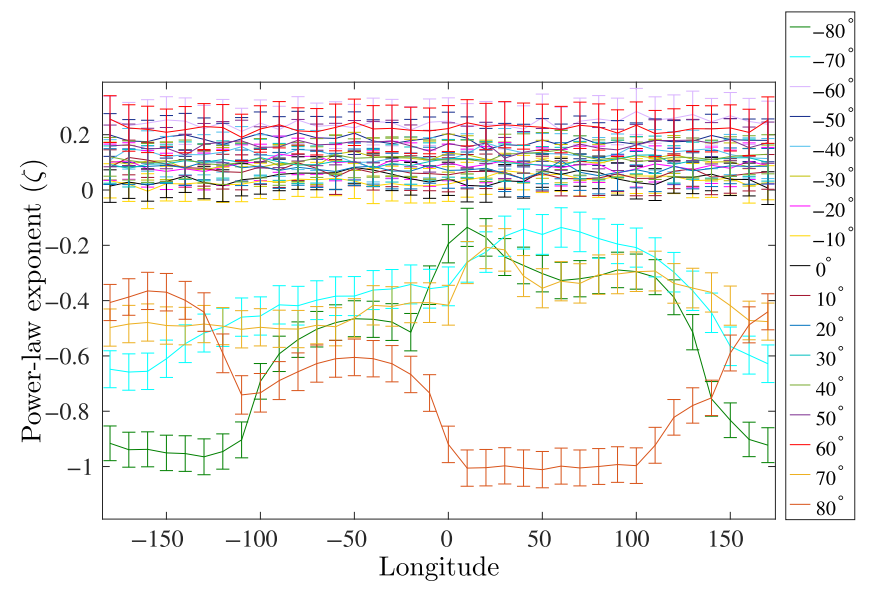

FIG. 14. Power-law exponent at intermediate frequencies for the direct radiation spectra. The power-law exponent is plotted against the longitude for each latitude. Other details are the same as in Fig. 13.

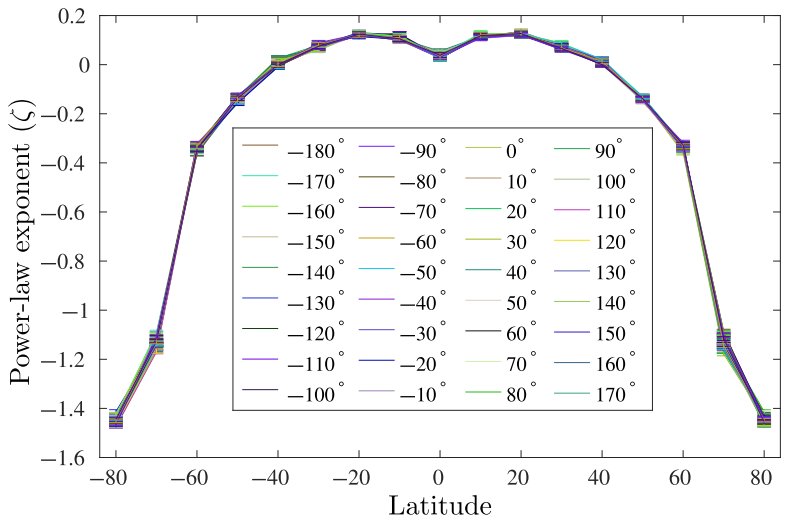

FIG. 15. Dependence of the power-law exponent at high frequencies for the direct radiation spectra. Other details are the same as in Fig. 13.

the clear-sky spectrum (without clouds) already possesses a power-law dependence, which we consider exclusively here. The global average computed by both methods is calculated synchronously in real time, i.e., the different irradiance intensities at different locations due to time lags are taken into account. Such an average includes temporal correlations that exist in real-world data. Considering timedelayed intensities would be relevant if a storage mechanism was used and the signals were combined at a certain time. However, this is not the situation we consider here.

In the first method, an average is calculated using CSMTL1 data for the year 2015 for four locations in an arbitrarily selected latitudinal range $\left(29^{\circ}-32^{\circ}\right)$ and at $34^{\circ}$ longitude where the spectral decay is minimal at low frequencies (Fig. 5) and, in fact, increases at high frequencies (Fig. 6) for both radiation components (see Fig. 17 for the power spectra at the abovementioned locations). The power spectra of the abovementioned latitudinally averaged radiation components are depicted in Fig. 7. On averaging the radiation at these four locations, the spectral

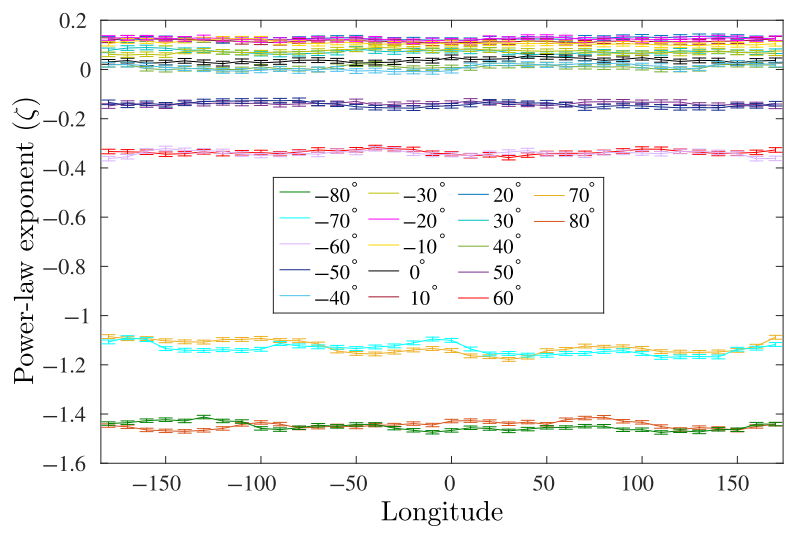

FIG. 16. Longitudinal dependence of the power-law exponent for the high-frequency direct radiation spectra. Other details are the same as in Fig. 14. 

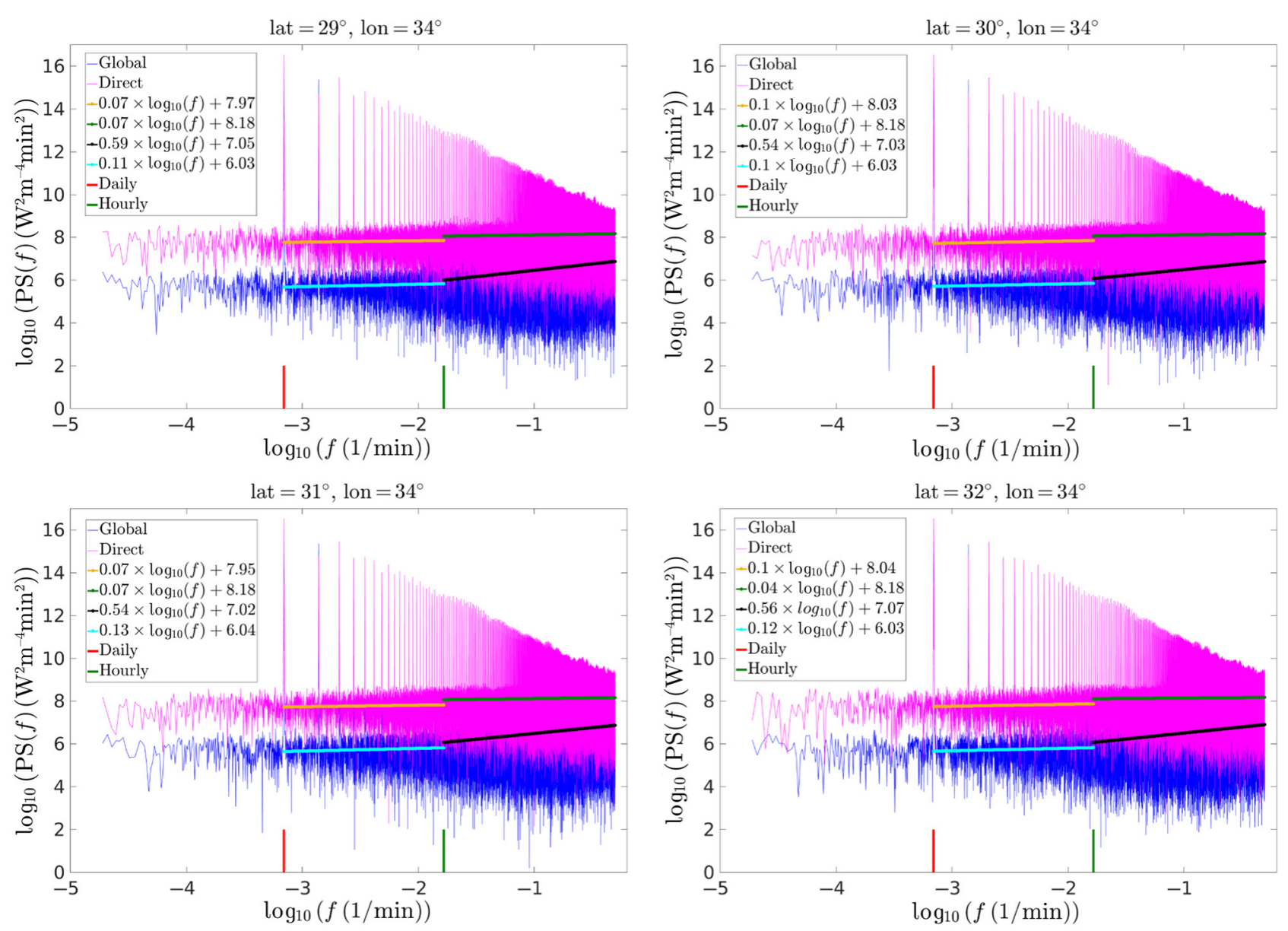

FIG. 17. Power spectra of the global and direct components at four different locations with the same longitude, $34^{\circ}$, and latitudes varying in steps of one degree from $29^{\circ}$ to $32^{\circ}$. We present the power spectra of each component and the power-law fit for the two different frequency ranges.

power-law exponent decreases only marginally for the global component in both frequency ranges, and, for the direct component, a marginal decrease is observed only at high frequencies. Overall, averaging over four locations at different latitudes (all close to $30^{\circ}$ ) but at a fixed longitude gives no significant spectral smoothing or modification for either radiation component. In the second averaging method, a similar scenario, at a fixed latitude $\left(30^{\circ}\right)$ and five longitudinal locations $\left(32^{\circ}-36^{\circ}\right)$ one degree apart, yields similar results (see Fig. 18).

Finally, we perform a global average of CSMTL1 data for the year 2015 over all global locations at which we calculate the radiation, which include 36 longitudinal values for each of 17 latitudinal values, yielding a total of 612 sampling points. We note that this type of averaging corresponds to the average radiation of areas of equal size centered at the location of the grid points. The spectra of the global and direct components are presented in Fig. 8. The weakening of the daily-frequency spectral peak owing to spatial averaging is readily apparent, and the difference in low- and high-frequency behavior observed at specific geographic locations is still captured in the global average. The spectral decay at intermediate frequencies steepens for both components [with the $95 \%$ confidence intervals of the power-law exponent being $(-3.24,-3.14)$ for the global component and $(-1.46,-1.38)$ for the direct component], whereas the high-frequency-range spectra remain flat [with the $95 \%$ confidence intervals of the power-law exponent being $(-0.24,-0.20)$ and $(-0.11,-0.09)$ for the global and direct components, respectively]. Note that the peak around a frequency of 1/40 min stems from the averaging procedure, which is based on locations separated by $10^{\circ}$ in longitude. The $10^{\circ}$ separation implies a $40-\mathrm{min}$ delay between the daylight in the different locations and, therefore, this peak and its higher harmonics appear in the spectra.

\section{DISCUSSION}

Having presented the results, we now explain the solarradiation spectrum starting from a simple temporally periodic signal, incremental additions of complex features 

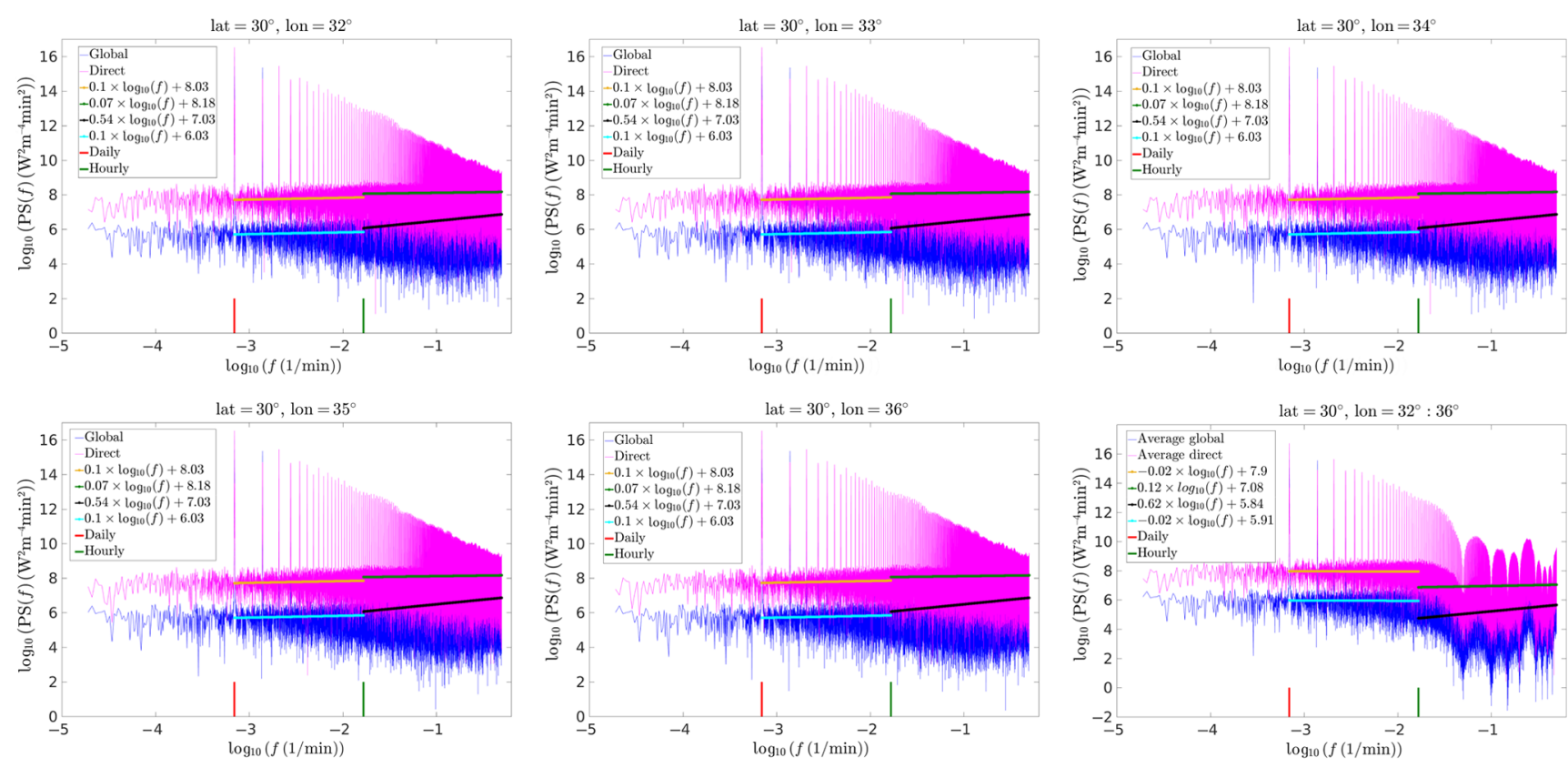

FIG. 18. Power spectra of the global and direct components at five different locations with the same latitude, $30^{\circ}$, and longitudes varying in steps of one degree from $32^{\circ}$ to $36^{\circ}$. We present the power spectra of each component and the power-law fit for the two different frequency ranges. The bottom right panel presents the same information for the radiation averaged over these five locations.

to which reveal the minimal ingredients that explain the primary characteristics of the radiation spectra, viz. their power-law dependence, and their intermediate- and highfrequency behavior. First, we generate a year-long time series with a 1-min time step for a periodic signal that is always positive, $\cos \left(2 \pi f_{d} t\right)+1$, where $f_{d}$ is the daily frequency (1/day), and, as expected, its spectrum (denoted "Periodic positive signal" in Fig. 9) has a sharp peak at the daily frequency and some noise, which is essentially zero (note log scale of the figure). The second power spectrum shown in Fig. 9, denoted "Masked periodic signal: none," corresponds to the periodic signal $\cos \left(2 \pi f_{d} t\right)$ filtered such that negative values are set to zero, i.e., the signal is $\max \left[\cos \left(2 \pi f_{d} t\right), 0\right]$. This is the most basic attempt to describe a signal with a daily frequency and a "dark" period for half of each daily cycle. In this signal, both the amplitude and the width of the nonzero period remain constant. The spectrum of this simply masked signal is very similar to that of the "Periodic positive signal," with the main difference being additional peaks at frequencies $f>1 /$ day, which appear due to the introduction of the "dark" periods.

Next we add an incremental feature to vary the nonzero (daylight) period and the amplitude (for solar radiation, both quantities vary with season). We implement this by using empirical data for the daylight duration on different dates at $35^{\circ}$ latitude. The daylight duration is updated every day throughout the year-long time series. The positive periodic signal is modified by setting it to zero outside the daylight period and shifting it down such that the light starts from zero. This modification results in a daily variation of both the duration of the "on time" and its amplitude (the maximum value during the "on time"). This signal is denoted "Masked periodic signal: AW" in Fig. 9, and its spectrum is readily seen to vary from the "Periodic positive" and "Masked periodic: none" signals. In particular, both the power-law spectral dependence and the difference in low- and high-frequency behavior (showing different power-law exponents) come into sharp relief. To understand whether one needs variation of both the "on time" and its amplitude in order to obtain the power-law dependence, we consider a fourth signal. This signal is similar to the third signal ("Masked periodic signal: AW"), but we normalize the signal such that for each day, the maximum value is equal to one. This fourth signal is denoted "Masked periodic signal: W" in Fig. 9. One sees that the power spectrum again exhibits a power-law dependence and two different exponents in the two regions of the frequency. Moreover, the power-law exponent for high frequencies is similar to the one for "Masked periodic signal: AW" but different at intermediate frequencies. This helps establish the fact that signals that vary more slowly than the daily frequency, viz. the daylight duration, control the spectral behavior in the intermediate- and high-frequency ranges.

These toy signals demonstrate that the variation of daylight duration is sufficient to obtain a spectral power-law dependence, which, in turn, explains its latitudinal variation. In the absence of daylight-duration variation, the power spectrum shows peaks at the daily frequency and its 
higher harmonics, while at other frequencies, the amplitude is below the numerical precision. Armed with this understanding, it is worth exploring its implications in various contexts. Since studies of climatic and ecological processes have focused on the impact of solar radiation at the low-frequency (subdaily) end, spectra of relevant quantities in these fields at intermediate and high frequencies are lacking. Having established that the seasonal variation in daylight duration controls the observed behavior at intermediate frequencies, we posit the thesis that practitioners of climate and ecological sciences should pay attention to intermediate- and high-frequency variations and treat them on a par with subdaily frequencies.

Given that all renewable-energy generation methods reflect the variability inherent in their energy sources $[16$, $17,36,37]$, and since high to intermediate frequencies are of interest in renewable-energy production and transmission, several studies have reported spectra in this frequency range for solar photovoltaic power production [11-15,17, 18]. For instance, the photovoltaic-power spectrum at high frequencies $(0.001 \mathrm{~Hz}<f<0.05 \mathrm{~Hz})$ (note that the daily frequency approximately equals $10^{-5} \mathrm{~Hz}$ and the hourly frequency is approximately equal to $3 \times 10^{-3} \mathrm{~Hz}$ ) was shown to follow the famous Kolmogorov [38] power-law decay with an exponent of $-5 / 3$ at two different locations (Hawaii and Germany), and the $-5 / 3$ exponent was attributed to the turbulent advection of clouds above PV plants being reflected in the spectrum. Two sites in Arizona exhibited a power-law decay with a spectral exponent of -2.6 for solar power at frequencies in the range of approximately $10^{-6}-10^{-3} \mathrm{~Hz}$ [12]. Another study, using data from a power plant in Spain, found that the PV plant acted as a low-pass filter [14]. Namely, it was shown that the measured irradiance spectrum followed a -1.4 power-law decay, while the spectrum of the power produced followed the same power law at low frequencies $\left(\sim 10^{-6}-10^{-3} \mathrm{~Hz}\right)$ and a steeper decay with a power-law exponent of -3.4 at higher frequencies $\left(\sim 10^{-3}-10^{-1} \mathrm{~Hz}\right)$. It was also suggested that the frequency at which the steeper decay starts may be a function of the plant size. An analysis of the power spectra of several PV power plants in India showed different power-law exponents in the range from -3.12 to -2.46 and that connecting several plants in the same area together resulted in a steeper decay, limited by the decay of the largest power plant [17].

Our analysis shows that the solar-radiation spectrum already exhibits a robust power-law decay at intermediate and high frequencies even in the absence of extraneous factors such as turbidity and cloud occlusion. We therefore submit that while cloud occlusion certainly plays an important role in photovoltaic-power fluctuations, this role should not be considered in a vacuum, but rather together with the bare solar-radiation spectrum that a location experiences. This is particularly so because power fluctuations introduced by cloud passage act multiplicatively on the bare solar radiation. The spectra and their power-law exponents reported in the literature for photovoltaic power would be of little consequence unless compared against their counterparts for bare solar radiation at the same location.

The above results may find immediate potential use within the solar photovoltaic industry. Firstly, when considering potential locations for a solar PV plant, the general industry practice is to study the average radiation maps $[39,40]$. If, in addition, one employs the CSM to study the bare radiation power spectrum, this provides additional information about the basic variability (excluding environmental factors) to expect at that location. Since variability adds a cost to renewables that is absent or reduced for conventional power sources such as coal and nuclear [37], knowledge of bare radiation spectra would help in anticipating the minimum costs due to fluctuations. Secondly, after a solar PV plant is operational, comparing the spectral power-law exponents for the CSM versus measured power fluctuations allows one to develop a standard metric that is globally applicable. In fact, the pertinent baseline spectrum to compare against should be the measured spectrum containing all environmental effects and not the CSM spectrum containing the geographic dependence alone; such an analysis will be undertaken in the future. Finally, geographic smoothing has limited efficacy in solar photovoltaics, as we show above, and this is due to all global locations being strongly correlated through a common source, viz. the Sun. Any attempts to mitigate or manage fluctuations at the grid level should explore engineering solutions.

\section{CONCLUSION}

In this paper, we analyze the fluctuations in solar radiation by studying the power spectra of its global and direct components. We show that a power-law dependence appears in both measured and calculated solar radiation and is not limited to the previously reported power-law dependence of the spectra of solar power generation. The exponent of the power law is used to characterize the geographic dependence of the radiation spectra in the range of frequencies from $1 /(2 \mathrm{~min})$ to $1 / \mathrm{yr}$. Using a simple toy model, we explained how the power-law spectrum emerges from the seasonal modulation of the diurnal signal. It is important to note that the power law shows a geographical dependence even for the calculated radiation (clear-sky model), which implies that this is not an effect of atmospheric processes such as clouds and turbidity.

The power-law exponent, which dictates the relative importance of fast fluctuations vis-à-vis the slower variation of solar intensity, can help in designing photovoltaic power plants. To ensure a smooth and continuous supply, the fluctuations have to be compensated for through 
engineered methods, be they other energy production mechanisms or, alternatively, storage devices. Naturally, locations with a smaller contribution from fast fluctuations are preferable. Understanding the geographic dependence of the clear-sky radiation is the first step toward practical modeling of photovoltaic power generation. The subsequent steps should involve the proper characterization of atmospheric effects, which include clouds and turbidity. The temporal correlations of these effects are expected to affect the power-law exponent of the fluctuations in the measured radiation. The last step involves relating the measured radiation to the actual production. Obviously, this relation is expected to have a geographical dependence because of the multiplicative nature of the atmospheric effects on the clear-sky radiation and, in turn, the effect of the photovoltaic devices on the incident radiation.

Geographical smoothing is shown to be limited for solar radiation due to global correlations, as opposed to the wind correlation length, which is of the order of hundreds of kilometers [36,37]. The above results hold for the clear-sky radiation, while turbidity and clouds are expected to have the correlation length of atmospheric processes. However, due to their multiplicative effect on the clear-sky radiation, the global correlation is not expected to disappear. Quantifying this latter effect is left for future studies.

Finally, we posit two theses: Firstly, experts in climate science and ecology should consider processes controlled by solar radiation at frequencies higher than the daily frequency because their character is determined by the variation at subdaily frequencies. Secondly, in research studies of photovoltaic-power fluctuations, one should consider comparing the measured photovoltaic-power spectra against bare solar-radiation spectra to better understand the role of clouds and how they change the spectral character.

\section{ACKNOWLEDGMENTS}

We thank Mr. Shlomo Kabalo for organizing and providing the measurement data from Sede Boqer. M.M.B. was funded by the Nonlinear and Non-equilibrium Physics Unit, OIST Graduate University. M.M.B. gratefully acknowledges generous hosting by Professor S. Sengupta at TCIS, TIFR Hyderabad while preparing this manuscript.

\section{APPENDIX A: GLOBAL-RADIATION-COMPONENT SPECTRUM}

Here we share additional data in support of claims made in the main text. Figure 10 presents the power spectra of the global and direct-components of solar radiation measured in Sede Boqer in year 2012. The power-law fits are similar to those measured for year 2009 and presented in Fig. 1.
Figure 11 presents the power-law dependence at intermediate-frequency range (1/day - 1 /hour) versus the longitude at various latitudes. It mirrors the data presented in Fig. 4 for same quantity plotted against latitude.

Figures 13 presents the high-frequency range power-law dependence versus the longitude. The information is same as that presented in Fig. 11 but versus the longitude.

\section{APPENDIX B: DIRECT-RADIATION-COMPONENT SPECTRUM}

Figure 13 shows that the power-law exponent for directradiation-component exhibits much stronger variation with longitude than the global-component as one approaches the poles. Moving away from the poles, it varies less than the global-component and remains nearly constant relative to the frequency, i.e. the power-law exponent is close to zero. This behavior is best illustrated in Fig. 14 where the power-law exponent at intermediate frequencies for direct-radiation-component is plotted versus longitude for various latitudes.

The high-frequency power-law exponent for the directradiation-component (Fig. 15) exhibits a similar trend as the global-component, but the values of the exponents are different. The value of the power-law exponent is close to zero away from the poles and decays less sharply than the global component near the poles. This trend is better illustrated in Fig. 16 where the longitudinal dependence of the power-law exponent is plotted for direct-radiationcomponent spectra against the longitude.

\section{APPENDIX C: SPATIAL AVERAGING OVER DIFFERENT LONGITUDES}

Figure 17 presents the power spectrum for both global and direct-radiation-components at the four different locations (latitudes $29^{\circ}-32^{\circ}$ in steps of $1^{\circ}$ and same longitude $34^{\circ}$ which were averaged to obtain result presented in Figs. 5 and 6.

Figure 18 presents the power spectrum for both global and direct-components at different locations (same latitude $30^{\circ}$ but longitudes varying in steps of one degree from $32^{\circ}$ to $36^{\circ}$ ). Averaging over these locations did not achieve significant smoothing as explained in main text.

[1] P. Alpert, P. Kishcha, Y. J. Kaufman, and R. Schwarzbard, Global dimming or local dimming?: Effect of urbanization on sunlight availability, Geophys. Res. Lett. 32, L17802 (2005).

[2] J. Lean and J. Rind, Climate forcing by changing solar radiation, J. Climate 11, 3069 (1998).

[3] A. T. Austin and L. Vivanco, Plant litter decomposition in a semi- arid ecosystem controlled by photodegradation, Nature 442, 555 (2006). 
[4] M. L. Bothwell, D. M. J. Sherbot, and C. M. Pollock, Ecosystem response to solar ultraviolet-b radiation: Influence of trophic-level interactions, Science 265, 97 (1994).

[5] T. M. Stoepler and J. T. Jill, Direct and indirect effects of light environment generate ecological trade-offs in herbivore performance and parasitism, Ecology 94, 2299 (2013).

[6] J. Ehleringer and I. Forseth, Solar tracking by plants, Science 210, 1094 (1980).

[7] R. Avissar, A statistical-dynamical approach to parameterize subgrid-scale land-surface heterogeneity in climate models, Survey. Geophys. 12, 155 (1991).

[8] J. M. Chen, J. Liu, J. Cihlar, and M. L. Goulden, Daily canopy photosynthesis model through temporal and spatial scaling for remote sensing applications, Ecol. Model. 124, 99 (1999).

[9] Y. Li, W. Han, T. Shinoda, C. Wang, R.-C. Lien, J. N. Moum, and J.-W. Wang, Effects of the diurnal cycle in solar radiation on the tropical indian ocean mixed layer variability during wintertime madden-julian oscillations, J. Geophys. Res.: Oceans 118, 4945 (2013).

[10] K. Klima, J. Apt, M. M. Bandi, P. Happy, C. Loutan, and R. Young, Geographic smoothing of solar photovoltaic electric power production in the Western USA, J. Renew. Sustain. Energy 10, 053504 (2018).

[11] J. S. G. Ehnberg and M. H. J. Bollen, Simulation of global solar radiation based on cloud observations, Solar Energy 78, 157 (2005).

[12] A. E. Curtright and J. Apt, The character of power output from utility-scale photovoltaic systems, Prog. Photovolt. Res. App. 16, 241 (2008).

[13] M. Lave and J. Kleissl, Solar variability of four sites across the state of colorado, Renewable Energy 35, 2867 (2010).

[14] J. Marcos, L. Marroyo, E. Lorenzo, D. Alvira, and E. Izco, From irradiance to output power fluctuations: the pv plant as a low pass filter, Prog. Photovolt. Res. App. 19, 505 (2011).

[15] M. Lave, J. Kleissl, and E. Arias-Castro, High-frequency irradiance fluctuations and geographic smoothing, Solar Energy 86, 2190 (2012).

[16] M. R. R. Tabar, M. Anvari, G. Lohmann, D. Heinemann, M. Wächter, P. Milan, E. Lorenz, and J. Peinke, Kolmogorov spectrum of renewable wind and solar power fluctuations, Euro. Phys. J. Spl. Topics 223, 2637 (2014).

[17] K. Klima and J. Apt, Geographic smoothing of solar PV: Results from Gujarat, Environ. Res. Lett. 10, 104001 (2015).

[18] M. Anvari, G. Lohmann, M. Wächter, P. Milan, E. Lorenz, D. Heinemann, M. R. R. Tabar, and J. Peinke, Short term fluctuations of wind and solar power systems, New J. Phys. 18, 063027 (2016).

[19] Richard Perez, Pierre Ineichen, Kathy Moore, Marek Kmiecik, Cyril Chain, Ray George, and Frank Vignola, A new operational model for satellite-derived irradiances: Description and validation, Solar Energy 73, 307 (2002).

[20] P. Ineichen and R. Perez, A new airmass independent formulation for the linke turbidity coefficient, Solar Energy 73, 151 (2002).

[21] A. Madanchi, M. Absalan, G. Lohmann, M. Anvari, and M. R. M. Tabar, Strong short-term non-linearity of solar irradiance fluctuations, Solar Energy 144, 1 (2017).
[22] C. J. T. Spitters, H. A. J. M. Toussaint, and J. Goudriaan, Separating the diffuse and direct component of global radiation and its implications for modeling canopy photosynthesis. Part I. Components of incoming radiation, Agric. For. Meteorol. 38, 217 (1986).

[23] Muhammad Iqbal, An Introduction to Solar Radiation (Academic Press, Ontario, Canada, 2012).

[24] A. A. El-Sebaii, F. S. Al-Hazmi, A. A. Al-Ghamdi, and S. J. Yaghmour, Global, direct and diffuse solar radiation on horizontal and tilted surfaces in Jeddah, Saudi Arabia, Appl. Energy 87, 568 (2010).

[25] D. H. W. Li, S. W. Lou, and J. C. Lam, An analysis of global, direct and diffuse solar radiation, Energy Procedia 75, 388 (2015).

[26] J. R. Petit, J. Jouzel, D. Raynaud, J.-M. Barnola, I. Basile, M. Benders, J. Chappellaz, M. Davis, G. Delaygue, M. Delmotte, V. M. Kotlyakov, M. Legrand, V. Y. Lipenkov, C. Lorius, C. Pépin, C. Ritz, E. Saltzman, and M. Stievenard, Climate and atmospheric history of the past 420,000 years from the Vostok ice core, Antarctica, Nature 399, 429 (1999).

[27] L. E. Lisiecki and M. E. Raymo, A pliocene-pleistocene stack of 57 globally distributed benthic $\delta^{18} \mathrm{O}$ records, Paleoceanography 20, PA1003 (2005).

[28] J. W. Kandelhardt, S. A. Zscheigner, E. Koscielny-Bunde, S. Havlin, A. Bunde, and H. E. Stanley, Multifractal detrended fluctuation analysis of nonstationary time series, Physica A 316, 87 (2002).

[29] R. Calif, F. G. Schmitt, and Y. Huang, Multifractal description of wind power fluctuations using arbitrary order hilbert spectral analysis, Physica A 392, 4106 (2013).

[30] R. Calif, F. G. Schmitt, Y. Huang, and T. Soubdhan, Intermittency study of high frequency global solar radiation sequences under a tropical climate, Solar Energy 98, 349 (2013).

[31] J. Remund, L. Wald, M. Lefèvre, T. Ranchin, and J. Page, in ISES Solar World Congress 2003 (International Solar Energy Society (ISES), Göteborg, Sweden, 2003), Vol. 400, p. 13.

[32] http://www.soda-is.com/eng/services/climat_free_eng.php\# c5-(retrieved July 17, 2012).

[33] M. Reno, C. Hansen, and J. Stein, Global Horizontal Irradiance Clear Sky Models: Implementation and Analysis, Tech. Rep. SAND2012-2389 (Sandia National Laboratories, Albuquerque, NM, USA, 2012).

[34] J. Apt, The spectrum of power from wind turbines, J. Power Sources 169, 369 (2007).

[35] P. Milan, M. Wächter, and J. Peinke, Turbulent Character of Wind Energy, Phys. Rev. Lett. 110, 138701 (2013).

[36] M. M. Bandi, Spectrum of Wind Power Fluctuations, Phys. Rev. Lett. 118, 028301 (2017).

[37] G. Bel, C. P. Connaughton, M. Toots, and M. M. Bandi, Grid-scale fluctuations and forecast error in wind power, New J. Phys. 18, 023015 (2016).

[38] A. N. Kolmogorov, The local structure of turbulence in incompressible viscous fluid for very large reynolds number, Dokl. Akad. Nauk SSSR 30, 299 (1941).

[39] https://globalsolaratlas.info/?c=41.15074,72.846488,2 (retrieved January 4, 2019).

[40] https://www.nrel.gov/gis/solar.html - (retrieved January 4, 2019). 